\title{
FOREWORD
}

\section{PAIN: FROM MOLECULES TO SUFFERING}

What is pain: a sensation, an experience, a symptom, or even a disease? We are all familiar with this deceptively simple term, but when it comes to description simplicity ends. No single definition seems to be able to encapsulate all the nuances of pain, which range from simple sensations to complex emotional experiences such as grief and suffering.

The essential element in pain is the activation of specialized high-threshold receptors to warn the organism of potential tissue damage. This sensory signal is followed, at least in humans, by a less well-defined but strong emotional experience - we are irresistably driven to stop the pain or escape from the stimulus that causes it. The complex combination of sensory-discriminative and affective-motivational elements in pain, and the role of cognitive processes in evaluating this information, limit our ability to understand how neurobiological signals are translated into pain. In this special focus issue, Vogt discusses how painful sensations and emotion interact in subregions of the cingulate gyrus - a crucial region for pain processing.

In the classical cartesian description, pain was considered to be a 'hard-wired' system in which noxious input was passively transmitted along sensory channels to the brain. However, it has been proved that this view is flawed - current neurobiological research has revealed that pain signalling involves a network of neurons, the functions and neurochemical profiles of which are constantly being modified by external and internal influences, with noxious stimuli themselves helping to drive this dynamic process. In addition, as discussed by Marchand et al., the immune system is an important modulator of pain processing.

Within a few decades, pain research has evolved

Troels Staehelin Jensen President Elect of the International Association for the Study of Pain (IASP), Danish Pain Research Center, Aarhus University Hospital, DK-8000 Aarhus C, Denmark.

e-mail: tsj@akhphd.au.dk for new and increased interactions between specialists such as neurobiologists, psychologists, clinicians and epidemiologists.

There are many important issues in pain research today. For example, why do some patients develop pain when others do not, despite exposure to the same damaging stimulus or disease? How is acute pain transformed into a chronic pain condition after a procedure, disease or injury, as occurs in $5-10 \%$ of patients? Presumably there are risk factors, or even specific genes that characterize patients who are at risk of chronic pain, and the ability to identify these factors or genes, and to understand how they predispose individuals to chronic pain, might help us to prevent or treat the condition. Another fascinating topic is the development of nociception and nociceptive circuits (as reviewed here by Fitzgerald). How does pain in a newborn infant or young child differ from the experience of an adult, and how should this influence the management of pain in children?

Despite the recent explosion of knowledge about the neurobiological processes that underlie pain, there is an enormous gap in the translation of this information into pain treatment. At present, even the most sophisticated pain therapies provide moderate to good pain relief for less than $30 \%$ of patients with chronic non-malignant types of pain. As Colloca and Benedetti discuss here, most treatments are also associated with a placebo effect. How does this occur, and can we make clinical use of it? There must also be additional, unidentified remedies for pain, and these need to be explored. For example, the explosion of research created by the discovery of endogenous modulatory systems in the 1970s has been followed by decades of silence. Perhaps it is time to revive some of these initial discoveries and see how they can be utilized in pain management. 